\title{
Correction to: Validation of a renal risk score in a cohort of ANCA-associated vasculitis patients with severe kidney damage
}

\author{
Juan M. Mejía-Vilet ${ }^{1}$ (D) - Eduardo Martín-Nares ${ }^{2}$ (D) - Mayra L. Cano-Verduzco ${ }^{3}$ (D) - Abril A. Pérez-Arias ${ }^{3}$ (D) \\ Manuel A. Sedano-Montoya ${ }^{2}$ (D) - Andrea Hinojosa-Azaola ${ }^{2}$ (C)
}

Published online: 13 March 2020

(C) International League of Associations for Rheumatology (ILAR) 2020

\section{Correction to: Clinical Rheumatology https://doi.org/10.1007/s10067-020-04936-5}

The publisher regret that the original published version of this article contained an error.

The sentence in the Introduction section original reading, "This clinicopathologic score includes three parameters (proportion of normal IF, and estimated glomerular filtration/ IF, and estimated glomerular filtration rate (eGFR) at the time of diagnosis)..." should be read as, "This clinicopathologic score includes three parameters (proportion of normal glomeruli, percentage of TA/IF, and estimated glomerular filtration rate [eGFR] at the time of diagnosis)..." [bold text used to highlight problem area]

The original article has been corrected.

Publisher's note Springer Nature remains neutral with regard to jurisdictional claims in published maps and institutional affiliations.

The online version of the original article can be found at https://doi.org/ 10.1007/s10067-020-04936-5

Andrea Hinojosa-Azaola

andreaha@yahoo.com

1 Department of Nephrology and Mineral Metabolism, Instituto Nacional de Ciencias Médicas y Nutrición Salvador Zubirán, Mexico, Mexico

2 Department of Immunology and Rheumatology, Instituto Nacional de Ciencias Médicas y Nutrición Salvador Zubirán, Vasco de Quiroga No. 15, Col. Sección XVI, Tlalpan, 14000 Mexico, CP, Mexico

3 Facultad de Medicina, Universidad Autónoma de Baja California campus Mexicali, Mexicali, Mexico 